\title{
On Steady MHD Thermally Radiating and Reacting Thermosolutal Viscous Flow through a Channel with Porous Medium
}

\author{
Promise Mebine ${ }^{1}$ and Rhoda H. Gumus ${ }^{2}$ \\ ${ }^{1}$ Department of Mathematics/Computer Science, Niger Delta University, Wilberforce Island, Nigeria \\ ${ }^{2}$ Department of Chemical/Petroleum Engineering, Niger Delta University, Wilberforce Island, Nigeria \\ Correspondence should be addressed to Promise Mebine, p.mebine@yahoo.com
}

Received 2 September 2009; Accepted 3 February 2010

Academic Editor: Michael Tom

Copyright ( 2010 P. Mebine and R. H. Gumus. This is an open access article distributed under the Creative Commons Attribution License, which permits unrestricted use, distribution, and reproduction in any medium, provided the original work is properly cited.

This paper investigates steady-state solutions to MHD thermally radiating and reacting thermosolutal viscous flow through a channel with porous medium. The reaction is assumed to be strongly exothermic under generalized Arrhenius kinetics, neglecting the consumption of the material. Approximate solutions are constructed for the governing nonlinear boundary value problem using WKBJ approximations. The results, which are discussed with the aid of the dimensionless parameters entering the problem, are seen to depend sensitively on the parameters.

\section{Introduction}

Thermosolutal or double diffusive convection is a transition process that involves concurrent heat and mass whenever there exist temperature and species concentration differences in a medium or between media, with one dependent on the other. This simultaneous occurrence of heat and mass transfer gradients is considered under conditions of technological and engineering importance. These are usually found in fluid-saturated porous media and are encountered in a wide range of thermal engineering applications such as in geothermal systems, oil extraction, ground water pollution, thermal insulation, heat exchangers, storage of nuclear wastes, packed bed catalytic reactors, atmospheric and oceanic circulation. Buoyancy induced flows are rife with references as provided in the text by Rubin and Atkinson [1].

The study of an electrically conducting fluid, which influences many natural and manmade flows, has many applications in engineering problems such as magnetohydrodynamics (MHDs) generators, plasma studies, nuclear reactors, geothermal energy extraction, and the boundary layer control in the field of aerodynamics. Sharma and Chaudhary [2] classified magnetic fields according to their various applications, namely, terrestrial magnetic field, 
which is maintained by fluid motion in the earths core, the solar magnetic field which generates sunspots and solar flares, and the galactic field which influences the formation of stars. A valuable review of the recent advances and applications of MHD-based microfluidic devices have been report by Qian and Bau [3], providing an extensive coverage. Many scientific researches also have transcended the MHD applications to geophysics and astrophysics due to high temperature phenomena or high-power radiation sources. Examples are found in combustion applications such as fires, furnaces, and IC engines; in nuclear reactions such as in the sun or in nuclear explosions as reported by Ghoshdastidar [4]; in compressors in ships and gas flares in the petroleum industries as provided by Abowei and Sikoki [5]. Peterson et al. [6] stated that where considerations of safety from waste-gases and emissions are concerned in petroleum industries, one of the performance parameters of interest is thermal radiation generated by the flare as a function of the waste-gas flowrate and composition. This implies that thermal radiation is very important in the study of combustion processes.

Combustion processes are very fast and exothermic reactions. Therefore, once the reaction is ignited the process proceeds very quickly and tends to be very nonisothermal. Hence, combustion processes release large amounts of energy, and they have many applications in the production of power, heat, and in incineration processes. Generally, combustion processes are complex because of the combination of complex kinetics, mass transfer control, and large temperature variations. For example, chemical reactions in high speed turbulent flows are accompanied with high temperatures and are of practical import. These are involved in hypersonic aircraft and re-entry vehicles. For nonisothermal chemical reactors, Schmidt [7] stated that they have nonlinearities that never occur in nonreacting systems. This explains the fact that in modeling chemically reactive combustible flows, the evolving equations are highly nonlinear. One feature of such equations as provided by Chung [8] is that the sensible enthalpy is coupled with the chemical species, which contributes to the heat source and diffusion of species interacting with temperature. Therefore, solutions to the evolving equations are mostly examined computationally. However, due to limited computational resources, the construction of approximate and ad hoc solutions to these nonlinear equations are in order.

The previous investigation on combustible flow of gas in a horizontal pipe in the presence of free convection with radiative heat transfer was carried out by Idowu and Adeoti [9]. The approximate analytical solutions of Idowu and Adeotu revealed the characteristics of the problem. It is the objective of this paper, therefore, to construct approximate solutions to the problem of MHD thermally radiating and reacting thermosolutal viscous flow through a channel with porous medium using a global approximation. These approximate solutions give a wider applicability in understanding the basic physics and chemistry of the problem, which are particularly important in industrial and technological fields.

In Section 2, the mathematical formulation of the problem and dimensionless forms of the governing equations are established. Solution method to these equations for the flow variables are briefly examined in Section 3. The results of the previous sections are discussed in Section 4. In Section 5, general concluding remarks of the results of the previous sections are given.

\section{Formulation of the Problem}

We consider the buoyancy induced steady flow in a porous medium bounded by two horizontal impermeable parallel walls. The lower wall which is on $y=-d / 2$ is maintained at a temperature $T=T_{1}$ and mass concentration $C=C_{1}$ and the upper wall which is on 
$y=d / 2$ is maintained at a temperature $T=T_{2}$ and mass concentration $C=C_{2}$, where $d$ is the distance between the upper and lower wall (see Figure 1). A chemical reaction takes place with a rate constant $k_{r}$ and a generalized Arrhenius activation energy is invoked in the mass concentration. A magnetic field of uniform strength $B_{0}$ is applied in the transverse direction of the channel and the induced magnetic field is neglected. With the aid of the Boussinesq approximation, the governing equations of the flow for an optically thin medium are then reduced to the following system of equations:

$$
\begin{gathered}
v \frac{d^{2} u}{d y^{2}}+g \beta_{T}\left(T-T_{0}\right)+g \beta_{C}\left(C-C_{0}\right)-\frac{\sigma_{c} B_{0}^{2}}{\rho_{0}} u-\frac{v}{k} u=0, \\
\alpha_{d} \frac{d^{2} T}{d y^{2}}-\frac{1}{\rho_{0} c_{p}} \frac{d q}{d y}=0 \\
D \frac{d^{2} C}{d y^{2}}-R(T) C=0 \\
\frac{d q}{d y}=4 \alpha \sigma\left(T^{4}-T_{0}^{4}\right) \\
R(T)=k_{r}^{2} T^{n} e^{-E_{A} / K_{B} T}
\end{gathered}
$$

where $u, T, y, \rho, \alpha_{d}, D, B_{0}, k, v, \sigma_{c}, \alpha, \sigma, \beta_{T}, \beta_{C}, R(T), k_{r}, n, E_{A}$, and $K_{B}$ represents flow, temperature, transverse coordinate, fluid density, thermal diffusivity, mass diffusivity, applied magnetic field strength, permeability of porous medium, kinematic viscosity, electric conductivity of the fluid, absorption coefficient or penetration depth, StefanBoltzmann constant, thermal expansion for temperature, solutal expansion for concentration, temperature dependent reaction rate coefficient, pre-exponential or frequency factor, an exponential constant, activation energy, and Boltzmann or universal gas constant. $g$ is the acceleration due to gravity.

The governing boundary conditions associated to (2.1a)-(2.1e) are

$$
\begin{aligned}
& u=0, \quad T=T_{1}, \quad C=C_{1} \quad \text { for } y=-\frac{d}{2}, \\
& u=0, \quad T=T_{2}, \quad C=C_{2} \quad \text { for } y=\frac{d}{2} .
\end{aligned}
$$

The radiative flux equation (2.1d) is highly nonlinear in $T$. However, when it is assumed that the temperature differences within the flow are sufficiently small, then the linear differential approximation of Cogley-Vincenti-Gilles equilibrium model [10] of the radiation flux becomes significant. In this case $T^{4}$ can be expressed as a linear function of temperature in Taylor series about $T_{0}$ neglecting higher-order terms. Thus,

$$
T^{4} \cong 4 T_{0}^{3} T-3 T_{0}^{4} .
$$

Therefore, $(2.1 \mathrm{~d})$ is now written as

$$
\frac{d q}{d y}=16 \alpha \sigma T_{0}^{3}\left(T-T_{0}\right)
$$




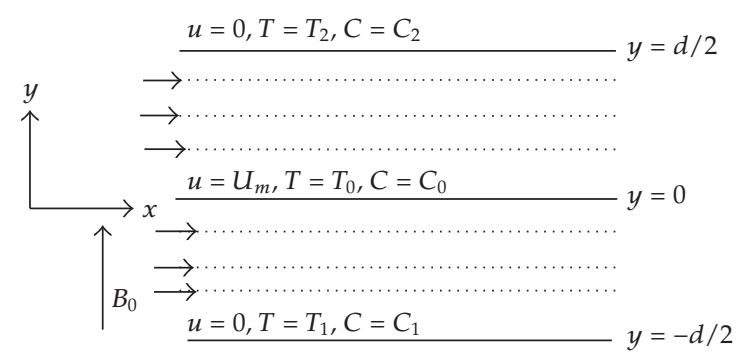

Figure 1: Physical model.

The functional form (2.1e) of the reaction-rate coefficient predicts a very strong dependence of reaction rates on temperature, and this fact is central in describing the complexities of chemical reactions. The factor $\exp \left(-E_{A} / K_{B} T\right)$ describes the probability of crossing a potential energy barrier between reactant and product molecules. In other words, $E_{A}$ is defined as this energy barrier (or threshold) that must be surmounted to enable the occurrence of the bond redistribution steps required to convert reactants into products. It is generally accepted that variations in the rate coefficient with temperature dependence using (2.1e) are with satisfactory accuracy for homogeneous processes with few exceptions as contained in the review by Angell et al. [11].

In order to facilitate the analysis, the following dimensionless variables and parameters are employed:

$$
\begin{gathered}
Y=\frac{y}{d}, \quad U=\frac{u}{U_{m}}, \quad \Theta=\frac{T}{T_{0}}, \quad \Phi=\frac{C}{C_{0}}, \\
M=\frac{\sigma_{c} B_{0}^{2} d^{2}}{\mu}, \quad G_{T}=\frac{\rho_{0} d^{2} \beta_{T} g T_{0}}{\mu U_{m}}, \quad G_{C}=\frac{\rho_{0} d^{2} \beta_{C} g C_{0}}{\mu U_{m}}, \\
X=\frac{k}{d^{2}}, \quad r^{2}=\frac{k_{r}^{2} d^{2} T_{0}^{n}}{D}, \quad E=\frac{E_{A}}{K_{B} T_{0}}, \quad N=\frac{16 \alpha \sigma T_{0}^{3} d^{2}}{\alpha_{d}},
\end{gathered}
$$

where $u=U_{m}$ is the mean flow.

Therefore, the dimensionless governing equations are

$$
\begin{gathered}
\frac{d^{2} U}{d Y^{2}}+G_{T}(\Theta-1)+G_{C} S(\Phi-1)-\left(M+\frac{1}{x}\right) U=0, \\
\frac{d^{2} \Theta}{d Y^{2}}-N(\Theta-1)=0, \\
\frac{d^{2} \Phi}{d Y^{2}}-r^{2} Q(Y) \Phi=0,
\end{gathered}
$$

where

$$
Q(Y)=\Theta^{n} e^{-E / \Theta}
$$


The boundary conditions are now

$$
\begin{gathered}
U=0, \quad \Theta=\Theta_{1}, \quad \Phi=\Phi_{1} \quad \text { for } Y=-\frac{1}{2} \\
U=0, \quad \Theta=\Theta_{2}, \quad \Phi=\Phi_{2} \quad \text { for } Y=\frac{1}{2} .
\end{gathered}
$$

The parameters entering the problem are $M$, magnetic parameter; $G_{T}$, thermal Grashof number; $G_{C}$, mass Grashof number; $x$, ratio of permeability of the porous medium and square of the characteristic length; $N$, radiation parameter; $E$, activation energy parameter; $n$, exponential constant and $r^{2}$, reaction-rate constant. The mathematical statement of the problem embodies the solution of (2.6a), (2.6b), and (2.6c) subject to (2.8a) and (2.8b).

\section{Solution of the Problem}

The posed problem (2.6a), (2.6b), and (2.6c) represents a system of coupled and nonlinear partial differential equations (PDEs). Exact and approximate analytical results of (2.6a), $(2.6 b)$, and (2.6c) subject to (2.8a), and (2.8b) are herein deduced. The energy (2.6b) is uncoupled from the momentum and concentration equation $((2.6 \mathrm{a})$ and (2.6c)). One can advance solution for the temperature variable $\Theta(Y)$ whereupon the solution of the flow $U(Y)$ and concentration $\Phi(Y)$ is then derived. Therefore, the solution for the temperature is given as follows:

$$
\begin{aligned}
\Theta(Y)= & \frac{1}{2 \cosh ((1 / 2) \sqrt{N}) \sinh ((1 / 2) \sqrt{N})} \\
& \times\left[\left(\Theta_{2}+\Theta_{1}\right) \sinh \left(\frac{1}{2} \sqrt{N}\right) \cosh (\sqrt{N} Y)-2 \sinh \left(\frac{1}{2} \sqrt{N}\right) \cosh (\sqrt{N} Y)\right. \\
& \left.\quad+\left(\Theta_{2}-\Theta_{1}\right) \cosh \left(\frac{1}{2} \sqrt{N}\right) \sinh (\sqrt{N} Y)+2 \cosh \left(\frac{1}{2} \sqrt{N}\right) \sinh \left(\frac{1}{2} \sqrt{N}\right)\right] .
\end{aligned}
$$

The concentration equation (2.6c) is nonlinear, and is not easily amenable to an exact solution. However, if we consider that the exponential constant $n$ and the activation energy $E$ are small, we can take $n=E=0$. In this case, the solution to (2.6c) becomes

$$
\begin{aligned}
\Phi(Y)= & \frac{1}{2 \cosh ((1 / 2) r) \sinh ((1 / 2) r)} \\
& \times\left[\left(\Phi_{2}+\Phi_{1}\right) \sinh \left(\frac{1}{2} r\right) \cosh (r Y)+\left(\Phi_{2}-\Phi_{1}\right) \cosh \left(\frac{1}{2} r\right) \sinh (r Y)\right] .
\end{aligned}
$$

Suppose that the constant exponent $n$ and the activation energy parameter $E$ are not necessarily small, then the ad hoc solution (3.2) is unacceptable. On the alternative, we assume that the chemical reaction rate $r$ is large and define it as

$$
r=\frac{1}{\epsilon}
$$




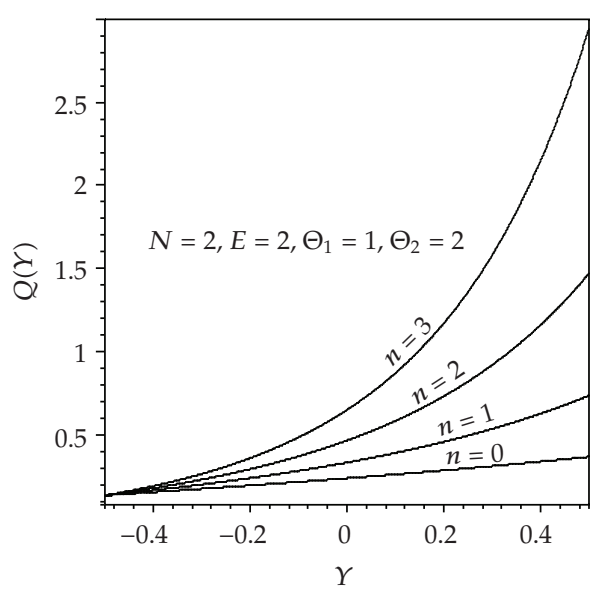

(a)

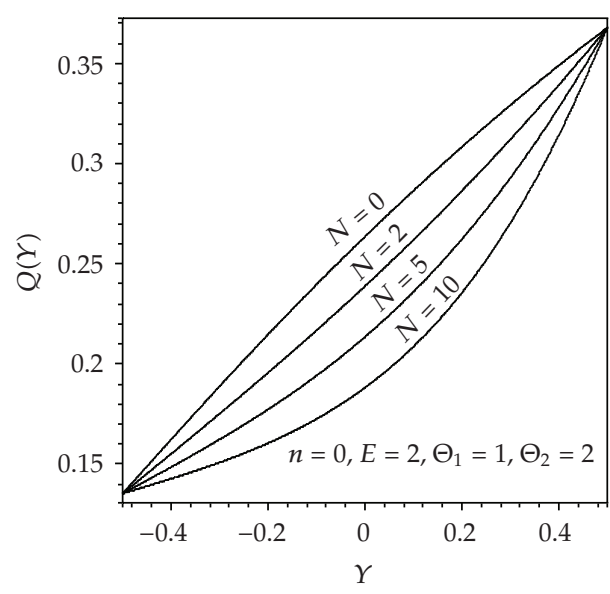

(b)

Figure 2: Potential function profiles for variations in the parameters: (a) Exponential constant, $n$; (b) Radiation parameter, $N$.

where $\epsilon$ is considered to be a small parameter. Equation (2.6c) now becomes

$$
\epsilon^{2} \frac{d^{2} \Phi}{d Y^{2}}-Q(Y) \Phi=0
$$

A powerful tool for obtaining a global approximate solution of a linear differential equation whose highest derivative is multiplied by a small parameter is the theory of WentzelKramers-Brillouin (WKB) or Wentzel-Kramers-Brillouin-Jeffrey (WKBJ), which is discussed in the texts by Stephenson and Radmore [12] and Hinch [13], respectively. An important application of the theory of WKBJ is in quantum mechanics, where it is used in the study of Schrödinger equation when particularly dealing with slowly varying potentials.

For the problem at hand, in applying the WKBJ approximation, only the eikonal and transport terms will be retained. Therefore, the general solution to (3.4) becomes

$$
\begin{aligned}
\Phi(Y)=Q(Y)^{-1 / 4}[ & \left\{\Phi_{2} Q\left(\frac{1}{2}\right)^{1 / 4}-\Phi_{1} Q\left(-\frac{1}{2}\right)^{1 / 4} \exp \left(-\frac{1}{\epsilon} \int_{-1 / 2}^{1 / 2} Q(s)^{1 / 2} d s\right)\right\} \\
& \left.\times \frac{\sinh \left(1 / \epsilon \int_{-1 / 2}^{Y} Q(s)^{1 / 2} d s\right)}{\sinh \left(1 / \epsilon \int_{-1 / 2}^{1 / 2} Q(s)^{1 / 2} d s\right)}+\Phi_{1} Q\left(-\frac{1}{2}\right)^{1 / 4} \exp \left(-\frac{1}{\epsilon} \int_{-1 / 2}^{Y} Q(s)^{1 / 2} d s\right)\right] .
\end{aligned}
$$

It is pertinent to remark here that the solution (3.5) will differ from the exact solution (if any) of the (3.4) by terms of order $\epsilon$ whenever $Q(Y) \neq 0$. However, if points exist where $Q(Y)=0$ (called the turning points of the equation), solution (3.5) will diverge at these points whereas a numerical integration of (3.4) will give a finite solution. In this case the WKBJ approximation exhibits the wrong behaviour close to the turning points. However, the way to handling solutions near turning points is a bit technical and is not presented in this paper. 
Now, having obtained the solutions for the temperature and concentration, we can proceed to the solution of the velocity. Therefore, the solution to (2.6a) becomes

$$
\begin{aligned}
U(Y)= & B_{1} \cosh (\lambda Y)+B_{2} \sinh (\lambda Y) \\
& -\left[\frac{G}{\lambda^{2}}+\frac{G_{T}}{\lambda} \int_{-1 / 2}^{Y} \sinh \{\lambda(Y-z)\} \Theta(z) d z+\frac{G_{C}}{\lambda} \int_{-1 / 2}^{Y} \sinh \{\lambda(Y-z)\} \Phi(z) d z\right],
\end{aligned}
$$

where $\lambda^{2}=1 / \chi+M, G=G_{T}+G_{C}$, and $B_{1}$ and $B_{2}$ are integration constants which are given by the following relations:

$$
\begin{aligned}
& B_{1}=\frac{1}{2 \cosh ((1 / 2) \lambda)}\left[\frac{2 G}{\lambda^{2}}+\frac{G_{T}}{\lambda} \int_{-1 / 2}^{1 / 2} \sinh \left\{\lambda\left(\frac{1}{2}-z\right)\right\} \Theta(z) d z\right. \\
& \left.+\frac{G_{C}}{\lambda} \int_{-1 / 2}^{1 / 2} \sinh \left\{\lambda\left(\frac{1}{2}-z\right)\right\} \Phi(z) d z\right] \\
& B_{2}=\frac{B_{1} \cosh ((1 / 2) \lambda)}{\sinh ((1 / 2) \lambda)}-\frac{G}{\lambda^{2} \sinh ((1 / 2) \lambda)}
\end{aligned}
$$

This completes the solutions of the problem.

\section{Discussion}

The potential function (2.7) together with the solutions (3.2), (3.5), and (3.6) make it possible to investigate quantitatively the manifestation of the effects of the various parameters entering the problem.

An understanding of the factors that control the potential function (2.7) that are relating to combustible materials is of fundamental importance in many industrial processes. Therefore, we begin the discussion with the potential function by investigating the effects of the exponential constant, $n$ and the radiation parameter, $N$. The potential function profiles are presented in Figure 2. It is observed in Figure 2(a) that for a given $N$ and activation energy parameter, $E$, the potential function increases with an increase in the exponential constant, $n$. On the other hand, Figure 2(b) depicts that for a given $n$ and $E$, an increase in the radiation parameter, $N$ sags the potential function, which appears to curve inwards.

We also considered the effect of the radiation parameter, $N$ on the temperature solution (3.1). From Figure 3, it is observed that an increase in the radiation parameter causes the temperature to move towards $Y=1 / 2$.

Now, the ad hoc concentration solution (3.2) depends only on the reaction-rate constant, $r$. Figure 4 demonstrates that the reaction-rate constant sags the concentration towards $Y=1 / 2$, just like what the radiation parameter does to the temperature.

In most of combustion calculations, there are several hundreds of reactions that could be considered. However, due to limited computational resources, it is customary to select only important reaction mechanisms, neglecting those that are less important (i.e., those reactions whose rates cannot be measured). Preliminary investigations in this work revealed that for $n \geq 1, Q(Y)=0$ gives turning points, where solution (3.5) at these points behaves 


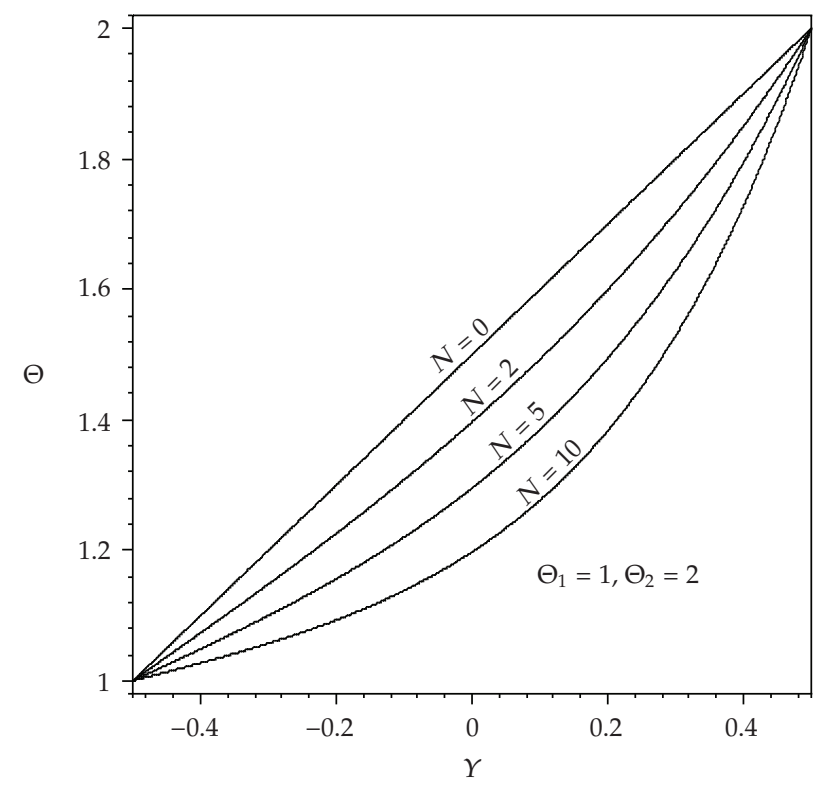

Figure 3: Temperature profiles for variations in the radiation parameter, $N$.

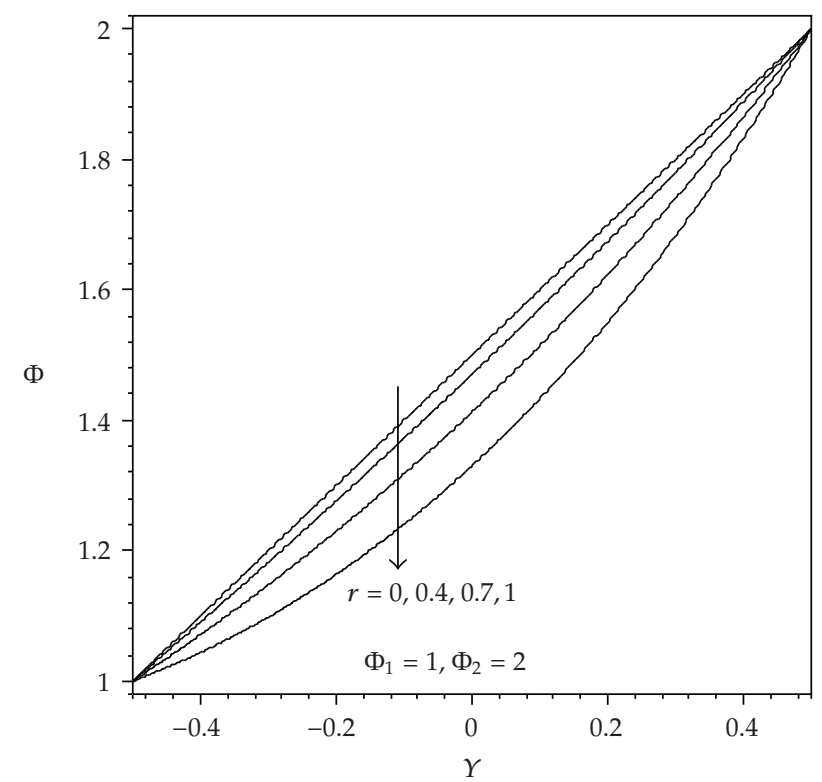

Figure 4: Concentration profiles as a function of $Y$ for variations in the reaction-rate constant, $r$.

wrongly. In fact, at these points, the computations using a Computer Symbolic Algebra Package (MAPLE) in a Macintosh Pentium 4 Machine shuts down the kernel. This means that the available memory is completely used up and MAPLE cannot continue without allocating more memory. Therefore, for the purpose of illustration, we consider the global system of hydrogen and oxygen:

$$
\mathrm{H}_{2}+\mathrm{O}_{2}=\mathrm{OH}+\mathrm{OH}
$$




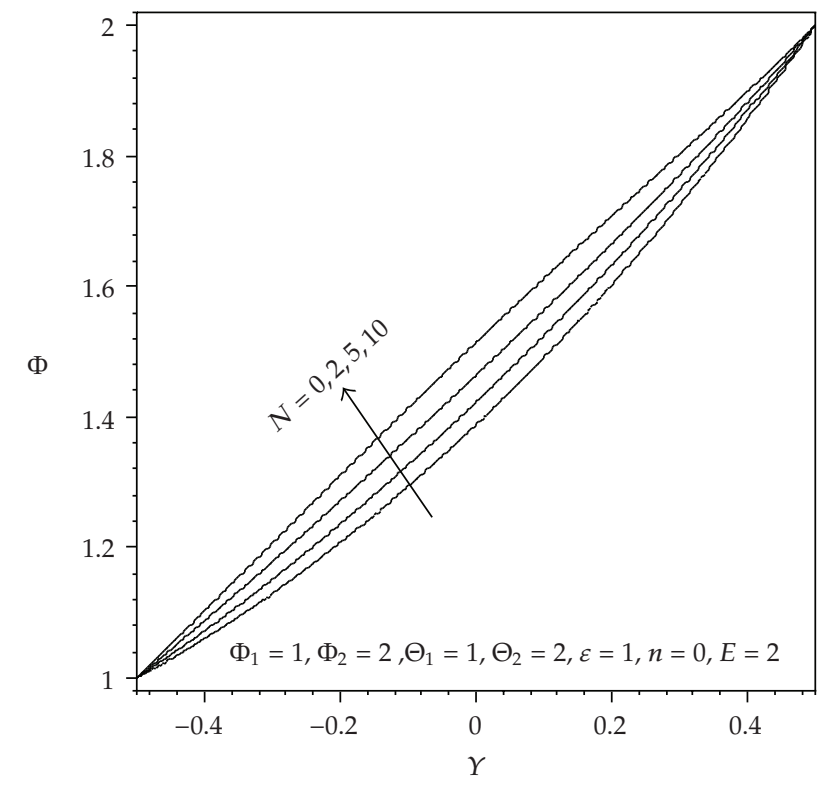

Figure 5: Concentration profiles as a function of $Y$ for variations in the radiation parameter, $N$.

as one example with $n=0$. Such information is available from existing literatures and are discussed extensively by Schmidt [7] and Chung [8]. Other example applications that take $n=0$ include kinetics of solid state reactions (mainly ionic crystals) as provided by Galwey and Brown [14], and deterioration and cooking of foods as examined in the article by Petrou et al. [15].

The WKBJ approximate concentration solution (3.5) is with the effect of the radiation parameter, $N$ via the temperature solution (3.1). Figure 5 results from the solution (3.5), and it shows that an increase in the radiation parameter causes the concentration to move towards $\Phi_{2}$.

Next, we examine the contributions of the temperature and concentration solutions to the velocity solution (3.6). Firstly, we consider the effect of the ad hoc concentration solution (3.2). Figures 6(a), 6(b), and 6(c) demonstrate the velocity profiles for different values of radiation parameter, $N$, magnetic-porosity parameter, $\lambda$ and reaction-rate constant, $r$, respectively, due to the ad hoc concentration solution. The maximum velocity is somewhere at the centre of the channel and tilts towards the right of the channel. It is evident that an increase in radiation, magnetic-porosity, and reaction-rate constant, reduces the velocity. For the radiation, it is so because it reduces the temperature, and the decrease in temperature changes the energy content of the reactants and the frequency of collisions, which affects the rate of a reaction. The application of the transverse magnetic field plays the role of a resistive type force (Lorentz force) similar to drag force (that acts in the opposite direction of the fluid motion), which tends to resist the flow thereby reducing its velocity. On the other hand, increasing $X$, physically indicates that the presence of a porous medium increases the resistance to flow and hence when $x=\infty$, the effect of porosity vanishes, which implies that the velocity would become greater in the flow field. One factor that affects the rate of a reaction is the concentration of reactants, which changes the frequency of collisions. In this case, the reaction-rate constant reduces the concentration, and hence the concentration in turn reduces the flow. This is depicted in Figure 6(c). 


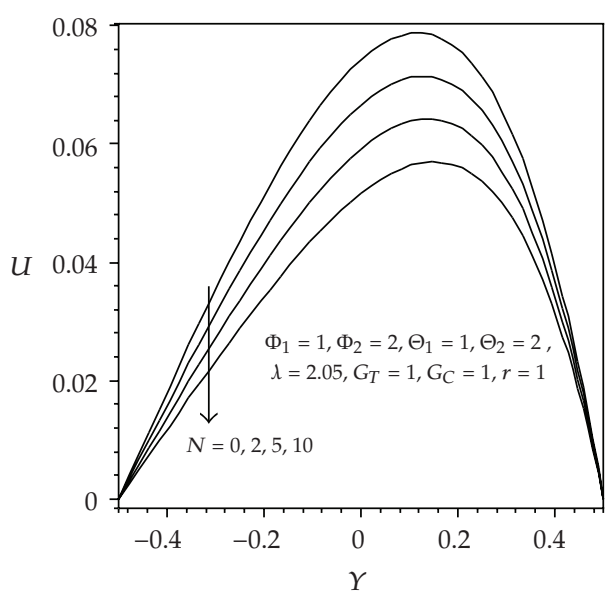

(a)

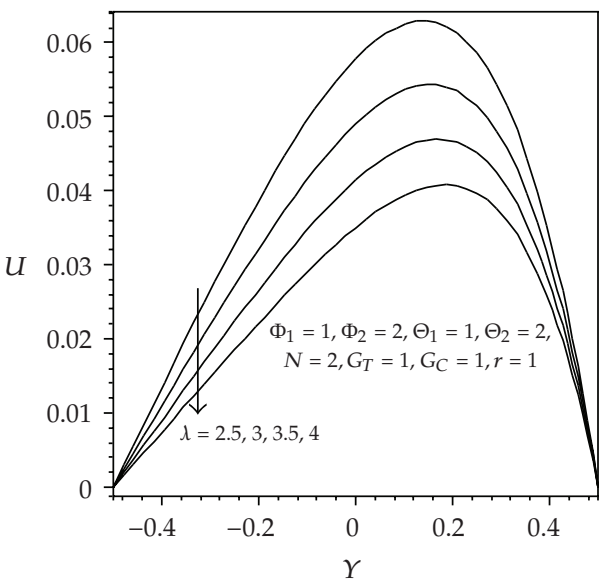

(b)

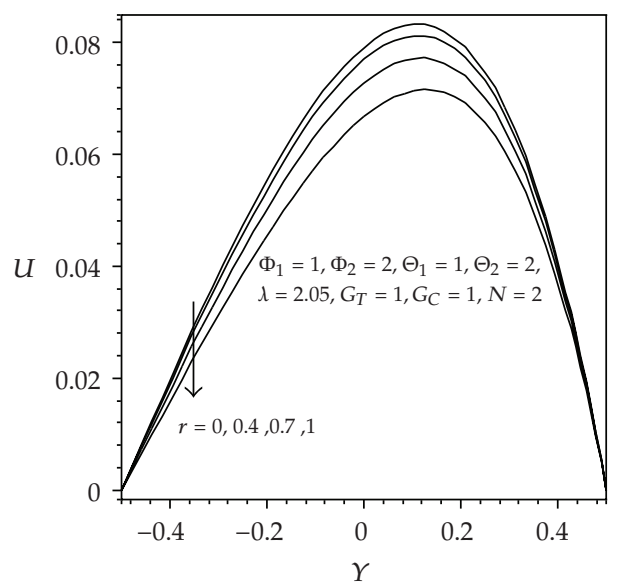

(c)

Figure 6: Velocity profiles as a function of $Y$ for variations in the parameters: (a) Radiation, $N$; (b) magnetic-porosity, $r$; (c) reaction-rate, $r$.

Secondly, we explore the effect of the WKBJ approximate concentration solution (3.2) on the velocity solution (3.6). It was evident that the solution replicates the patterns (i.e., Figure 6 for the ad hoc concentration solution) shown by radiation parameter, $N$, magneticporosity parameter, $\lambda$ and reaction-rate constant, $r$, when $n=0$ and $E=2$. However, significant differences between the two solutions were only seen at about the centre of the channel. Therefore, tabular representations (see Table 1) are herein made at $Y=0$ for the comparison.

In each of the columns (1-3) in the table, (3.2) and (3.5), refer to the velocity solution due to the concentration solution (3.2) and (3.5), respectively. Therefore, it can be seen from Table 1 that the velocity solution (3.2) and (3.5) at the centre of the channel, increases with a decrease in the value of $N$ and $\lambda$, with the values of the velocity solution (3.5) appearing dominantly greater than those for (3.2). On the other hand, an increase in $r$ causes a decrease in the velocity solution (3.2) and (3.5). Here for $0.0 \leq r \leq 0.4$, the values of (3.2) are greater than the values of (3.5), and vice versa for $0.7 \leq r \leq 1.0$. 
Table 1: Comparison of velocity solution (3.6) at the centre of the channel due to (3.2) and (3.5), respectively.

\begin{tabular}{|c|c|c|c|c|c|c|c|c|}
\hline \multicolumn{3}{|c|}{$(1)$} & \multicolumn{3}{|c|}{$(2)$} & \multicolumn{3}{|c|}{ (3) } \\
\hline$N$ & $\begin{array}{c}\text { Velocity } \\
\text { equation } \\
(3.2)\end{array}$ & $\begin{array}{c}\text { Velocity } \\
\text { equation } \\
(3.5)\end{array}$ & $\lambda$ & $\begin{array}{c}\text { Velocity } \\
\text { equation } \\
(3.2)\end{array}$ & $\begin{array}{c}\text { Velocity } \\
\text { equation } \\
(3.5)\end{array}$ & $r$ & $\begin{array}{c}\text { Velocity } \\
\text { equation } \\
(3.2)\end{array}$ & $\begin{array}{c}\text { Velocity } \\
\text { equation } \\
(3.5)\end{array}$ \\
\hline 0 & 0.0742590085 & 0.0784487847 & 2.5 & 0.0578335848 & 0.0636934325 & 0.0 & 0.0791013367 & 0.0765976954 \\
\hline 2 & 0.0666834061 & 0.0733996050 & 3.0 & 0.0489935405 & 0.0539958380 & 0.4 & 0.0769449694 & 0.0760755165 \\
\hline 5 & 0.0591602527 & 0.0688520731 & 3.5 & 0.0414079865 & 0.0456719438 & 0.7 & 0.0727141684 & 0.0750122273 \\
\hline 10 & 0.0517051176 & 0.0649783577 & 4.0 & 0.0350527304 & 0.0386955124 & 1.0 & 0.0666834061 & 0.0733996049 \\
\hline
\end{tabular}

\section{Concluding Remarks}

It is hoped that the present investigation may serve as toolkits for numerical experimentations. It is noted here that the efficient computation of thermal radiation effect with strongly exothermic reaction under generalized Arrhenius kinetics is essential for the design and analysis of industrial thermal systems, such as furnaces, boilers, burners, nuclear power plants, combustion products (such as $\mathrm{H}_{2} \mathrm{O}$ and $\mathrm{CO}_{2}$ ), and gas turbines. The results of the problem are also of great interest in geophysics in the study of interaction of the geomagnetic field with the fluid in the geothermal region.

\section{References}

[1] H. Rubin and J. Atkinson, Environmental Fluid Mechanics, Marcel Dekker, New York, NY, USA, 2001.

[2] P. K. Sharma and R. C. Chaudhary, "Magnetohydrodynamics effect on three-dimensional viscous incompressible flow between two horizontal parallel porous plates and heat transfer with periodic injection/suction," International Journal of Mathematics and Mathematical Sciences, vol. 2004, no. 62, pp. 3357-3368, 2004.

[3] S. Qian and H. H. Bau, "Magneto-hydrodynamics based microfluidics," Mechanics Research Communications, vol. 36, no. 1, pp. 10-21, 2009.

[4] P. S. Ghoshdastidar, Heat Transfer, Oxford University Press, Oxford, UK, 2004.

[5] J. F. N. Abowei and F. D. Sikoki, Water Pollution Management and Control, Doubletrust Publications, Port Harcourt, Nigeria, 2005.

[6] J. Peterson, N. Tuttle, H. Cooper, and C. Baukal, "Minimize facility flaring," Hydrocarbon Processing, vol. 86, no. 6, pp. 111-115, 2007.

[7] L. D. Schmidt, The Engineering of Chemical Reactions, Oxford University Press, Oxford, UK, 1998.

[8] T. J. Chung, Computational Fluid Dynamics, Cambridge University Press, Cambridge, UK, 2002.

[9] A. S. Idowu and O. A. Adeoti, "On the flow of combustible gas in a horizontal pipe," Research Journal of Applied Sciences, vol. 3, no. 8, pp. 539-544, 2008

[10] A. C. L. Cogley, W. G. Vincenti, and E. S. Gilles, “Differential approximation for radiative heat transfer in a nonlinear equations-grey gas near equilibrium," American Institute of Aeronautics and Astronautics Journal, vol. 6, pp. 551-553, 1968.

[11] C. A. Angell, K. L. Ngai, G. B. McKenna, P. F. McMillan, and S. W. Martin, “Relaxation in glassforming liquids and amorphous solids," Journal of Applied Physics, vol. 88, no. 6, pp. 3113-3157, 2000.

[12] G. Stephenson and P. M. Radmore, Advanced Mathematical Methods for Engineering and Science Students, Cambridge University Press, Cambridge, UK, 1990.

[13] E. J. Hinch, Perturbation Methods, Cambridge Texts in Applied Mathematics, Cambridge University Press, Cambridge, UK, 1991. 
[14] A. K. Galwey and M. E. Brown, "A theoretical justification for the application of the Arrhenius equation to kinetics of solid state reactions (mainly ionic crystals)," Proceedings of the Royal Society A, vol. 450, no. 1940, pp. 501-512, 1995.

[15] A. L. Petrou, M. Roulia, and K. Tampouris, "The use of the Arrhenius equation in the study of deterioration and of cooking of foods-some scientific and pedagogic aspects," Research and Practice in Europe, vol. 3, no. 1, pp. 87-97, 2002. 


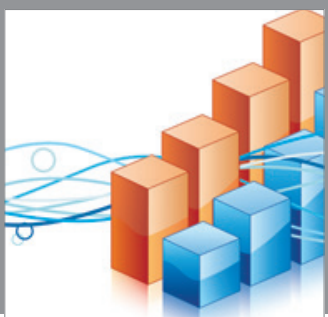

Advances in

Operations Research

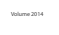

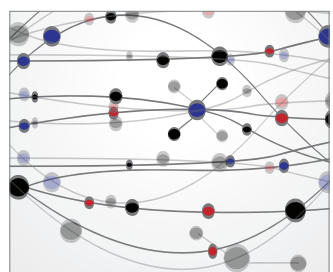

\section{The Scientific} World Journal
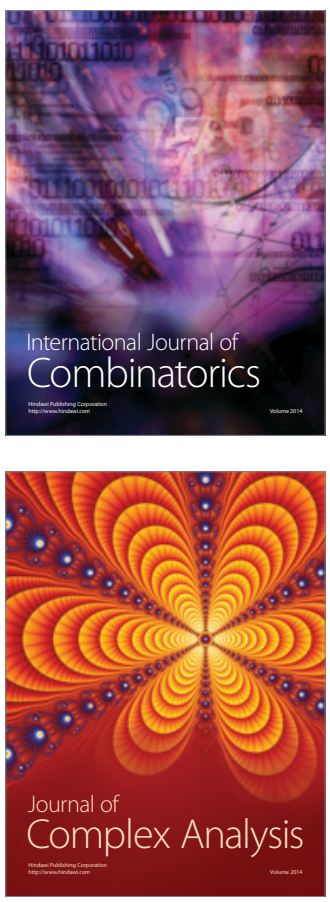

International Journal of

Mathematics and

Mathematical

Sciences
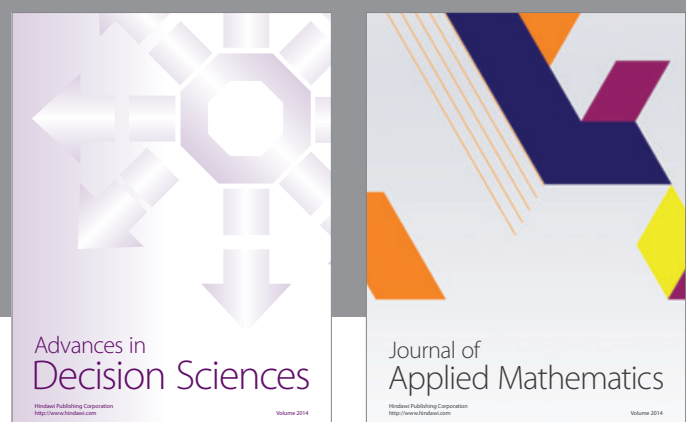

Journal of

Applied Mathematics
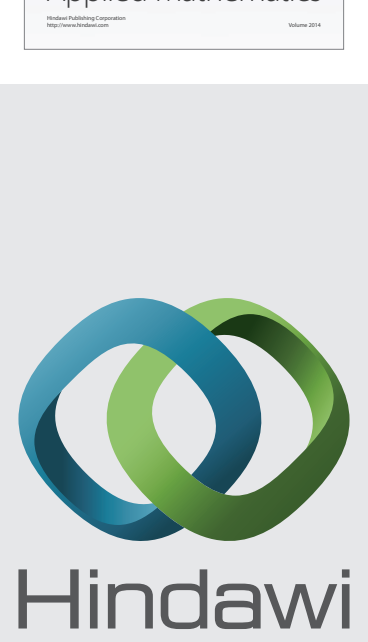

Submit your manuscripts at http://www.hindawi.com
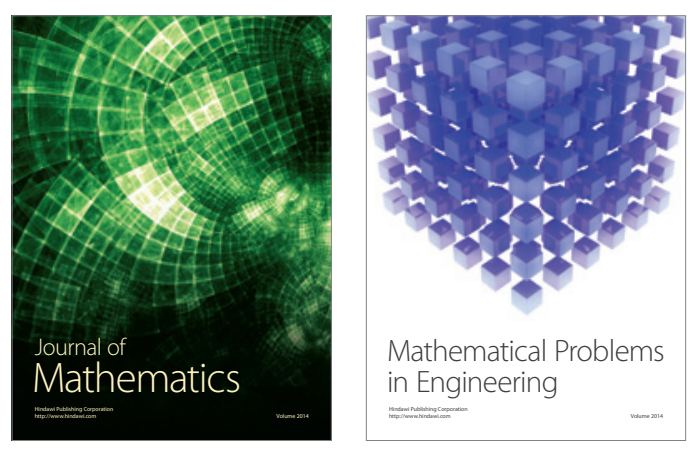

Mathematical Problems in Engineering
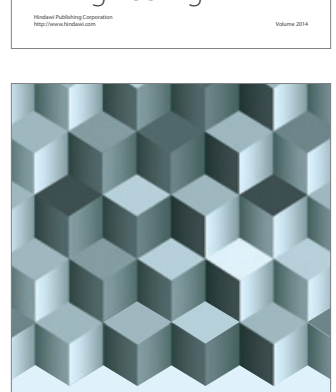

Journal of

Function Spaces
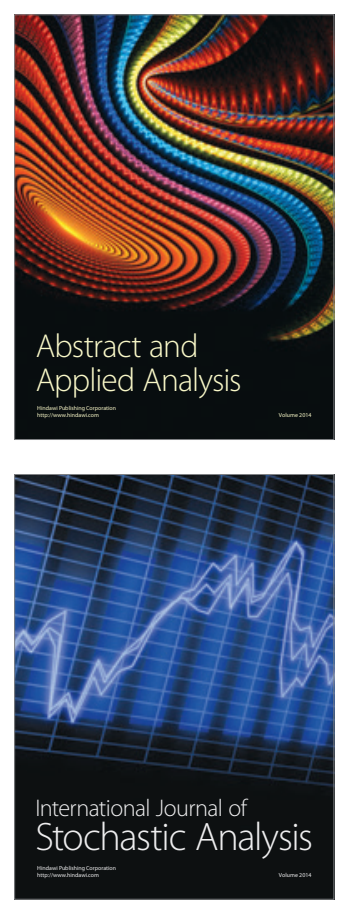

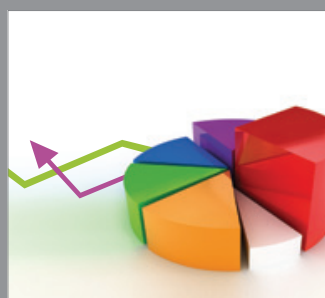

ournal of

Probability and Statistics

Promensencen
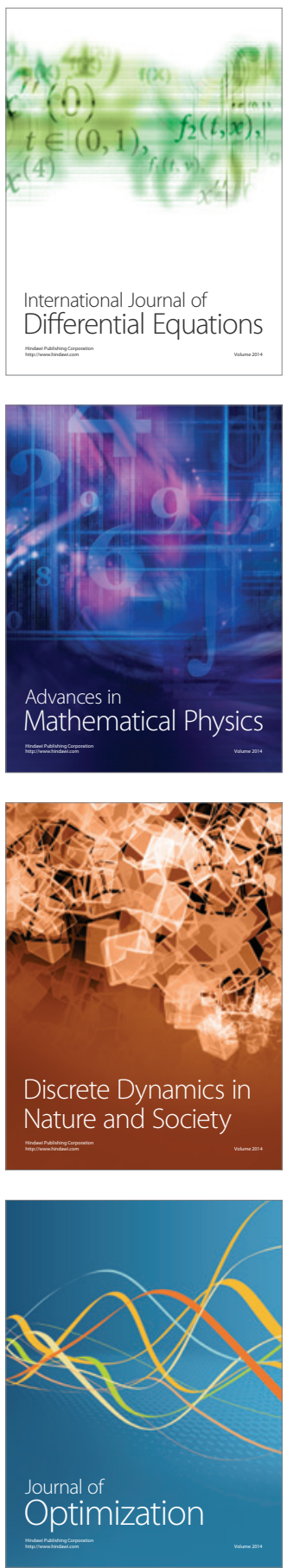